\title{
Photodynamic therapy of choroidal neovascularization with enlargement of the spot size to include the feeding complex
}

\author{
Ilias Georgalas \\ Alexandros A Rouvas \\ Dimitrios A Karagiannis \\ Athanasios I Kotsolis \\ loannis D Ladas \\ Department of Ophthalmology, \\ Medical School of Athens University, \\ Athens, Greece
}

\begin{abstract}
This is a case report of a 83-year-old man with choroidal neovascularization (CNV), due to age-related macular degeneration (AMD) in his right eye. Digital fluorescein (FA) and indocyanine green angiography (ICG) were performed, which disclosed predominantly classic subfoveal CNV and a dilated and tortuous feeding complex. The visual acuity was 20/800. Anti-vascular endothelial growth factor (anti-VEGF) treatment was suggested, however, the patient was not keen to receive an intraocular injection. Modified photodynamic therapy (PDT) with spot size enlarged, to include not only the CNV lesion but the feeding complex as well, was performed. Ten days after one session of PDT, ICG showed absence of leakage from the $\mathrm{CNV}$ and complete occlusion of the feeding complex. The visual acuity gradually improved to 20/100 and remained stable during the following 23 months. No evidence of CNV leakage was seen in the FA and ICG during the follow up period. Adjustment of the PDT spot size to include the detectable by ICG feeding complex might be an additional option in order to close the subfoveal CNV and might be considered as an alternative to intravitreal injection of antiVEGF in selected cases where anti-VEGF treatment is not available.
\end{abstract}

Keywords: age-related macular degeneration, choroidal neovascularization, photodynamic treatment, feeder vessel

\section{Introduction}

Photodynamic therapy (PDT) has been used in the recent past years as an effective treatment to prevent significant loss of vision in patients with choroidal neovascularization (CNV) secondary to age-related macular degeneration (AMD) especially when the CNV is classic or predominantly classic. ${ }^{1,2}$

Previous studies demonstrated that $22 \%-44 \%$ of patients with CNV lesions have demonstrable feeder vessels. ${ }^{3}$ The concept of indocyanine green angiography (ICG)-guided feeder vessel photocoagulation has demonstrated favorable results in neovascular AMD. ${ }^{4-6}$

We report a favourable result with cessation of leakage of a subfoveal CNV in a patient with exudative AMD treated with a single session of PDT when the choroidal complex was included all along in the treatment spot.

\section{Case report}

An 83-year-old Caucasian man with history of diabetes mellitus, high blood pressure, and a nonsmoker was referred to us with the diagnosis of exudative maculopathy due to AMD in his right eye. His best corrected visual acuity (BCVA) of the right eye was $20 / 800$.

The Topcon Imagenet 2000 Digital Imaging System with Imagenet 2000 (version 2.14) software and the TRC-50IA fundus camera was used to perform fluorescein 
(FA) and ICG angiography. FA angiography depicted a predominantly classic subfoveal $\mathrm{CNV}$ in the right eye of the patient (Figure 1). Further, ICG revealed a dilated and tortuous feeding complex of the CNV with prolonged course raised from the deeper choroidal layers filling early after dye administration (Figure 2).

Intravitreal anti-vascular endothelial growth factor (anti-VEGF) treatment was suggested to the patient, however, he adamantly refused to receive intraocular injections of any kind.

After a discussion underlining possible risks and benefits the patient decided to undergo photodynamic therapy instead of anti VEGF treatment. PDT was performed to treat the CNV according to the protocol of the treatment of AMD with PDT study. ${ }^{1,2}$ However the treatment spot size was enlarged to include in the treatment beam not only the CNV but also the feeding complex. Treatment was approved by the hospital Institutional Review Board.

Ten days after first session of PDT, ICG showed cessation of leakage of the CNV with complete occlusion of the feeder vessel (Figure 3). BCVA improved to 20/200. The patient did not attend his three months follow up appointment, as recommended. Six months post-PDT, FA and ICG (Figure 4A, B) showed cessation of the leakage of the previous subfoveal CNV. BCVA improved to 20/80. The FA and ICG images, as well as the BCVA remained stable up till today (23 months after PDT treatment).

\section{Discussion}

Anti-VEGF treatment has resulted in unprecedented visual and anatomic outcomes far outpacing other available treatments. ${ }^{7-9}$ Today physicians and patients can expect visual stabilization in most patients and visual improvement in

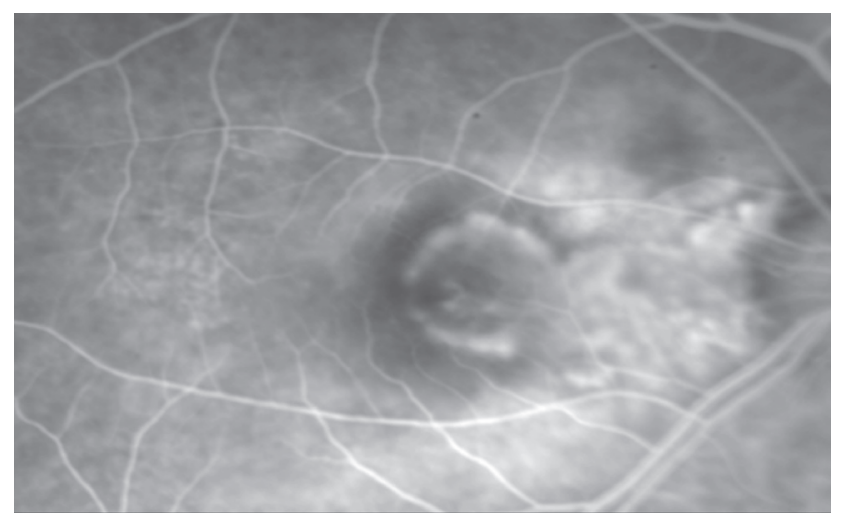

Figure I Before PDT, fluorescein angiography shows a predominantly classic subfoveal CNV.

Abbreviations: CNV, choroidal neovascularization; PDT, photodynamic therapy. many, particularly, if treatment is begun early in the course of the disease. PDT in combination with anti-VEGF have been also used for the treatment of CNV. ${ }^{10,11}$ However, similar to our patient, not all patients are happy to receive intraocular injections. Additionally, not all patients are able to afford the monthly treatment with anti-VEGF.

PDT has been proven to be beneficial in reducing the risk of severe visual loss in selected patients with CNV caused by AMD. Randomised clinical trials of verteporfin therapy have demonstrated that an average of 3-4 retreatments are needed to control the CNV due to AMD in the first year, decreasing to 2-3 in the second year. PDT provides temporary immediate occlusion of the CNV and absence of leakage in the FA after a single PDT. ${ }^{12}$ However studies with ICG have demonstrated in the great majority of cases the occlusion was not complete and the CNV remained open at least at the level of the feeder vessel. Schmidt-Erfurth and colleagues showed that feeder vessel occlusion after PDT is not complete and that the CNV complex remains patent at the level of $\mathrm{FV}$ in $50 \%$ of treated patients. ${ }^{3,12}$ Persistent feeder vessels were found to be the origin of newly developing neovascular proliferation. ${ }^{13}$

Occlusion of the feeder vessel, using modifications of the classic PDT treatment have been described in the recent years and the safety ${ }^{3}$ of extrafoveal PDT has been proven. ${ }^{3,4,14}$ Piermarocchi and colleagues reported 16 eyes that received direct laser photocoagulation of the feeder vessel after PDT and did not need any further photodynamic therapy. ${ }^{4}$ Staurenghi and colleagues reported favorable results in 10 patients where the PDT was combined with feeder vessel laser treatment in comparison to those from 10 patients who

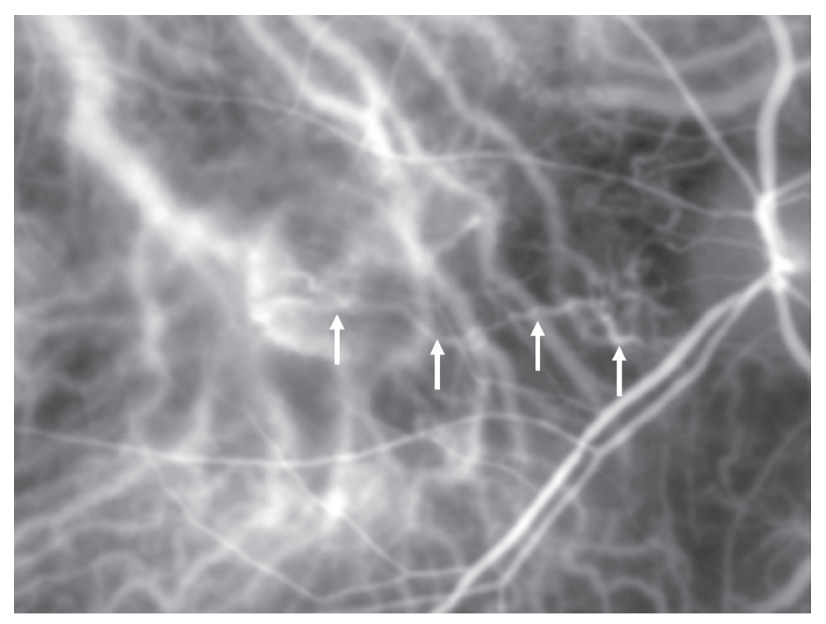

Figure 2 Before PDT, early-phase ICG illustrates a dilated and tortuous feeding complex (arrows) of the subfoveal CNV raised from a deeper choroidal layer. Abbreviations: CNV, choroidal neovascularization; ICG, indocyanine green angiography; PDT, photodynamic therapy. 


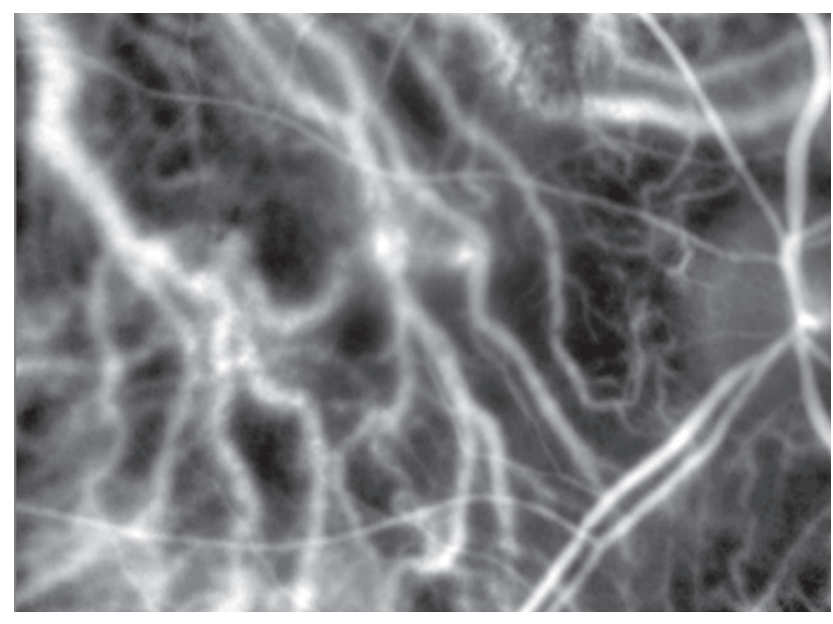

Figure 3 Ten days after PDT, early-phase ICG: Complete disappearance of the CNV and the choroidal feeder vessel.

Abbreviations: CNV, choroidal neovascularization; ICG, indocyanine green angiography; PDT, photodynamic therapy.

received treatment under the standard protocols of PDT. ${ }^{5}$ Sickenberg and colleagues described two patients with AMD who, apart from the PDT, received complementary feeder vessel treatment. ${ }^{14}$ In the first patient, the feeder vessel was occluded by minimal photocoagulation immediately after PDT treatment. The second patient was treated initially with a PDT spot covering the lesion, followed immediately by a small spot focused on the feeder vessel. In both cases subfoveal CNV was successfully occluded after PDT and some visual acuity was gained following the treatment.

In our patient, cessation of the leakage from the CNV was evident not only in the FA but also in the ICG, 10 days after a single session of PDT treatment when the feeding complex was included in the treatment spot. No evidence of recurrent $\mathrm{CNV}$ was seen in the FA or ICG during the follow up period. The visual acuity of our patient improved and remained unchanged for the following 23 months.

Of course, further research needs to be conducted in order to evaluate these findings. Data from one patient can not establish a cause-effect relationship between treatment and clinical outcome. However, this case highlights the synergetic association of PDT with treatment modifications targeting the feeding complex of the CNV. The potential benefit from a single PDT session with inclusion of the feeding complex in the PDT spot should be weighed against the detriment of treating a larger area of normal choroid and the effects of multiple PDT treatments to the normal choriocapillaries. The fact that PDT is a relatively inexpensive treatment modality should be also taken into consideration.

We have entered the era of anti-VEGF therapy in the treatment of $\mathrm{CNV}$ in patients with AMD. However in cases
A

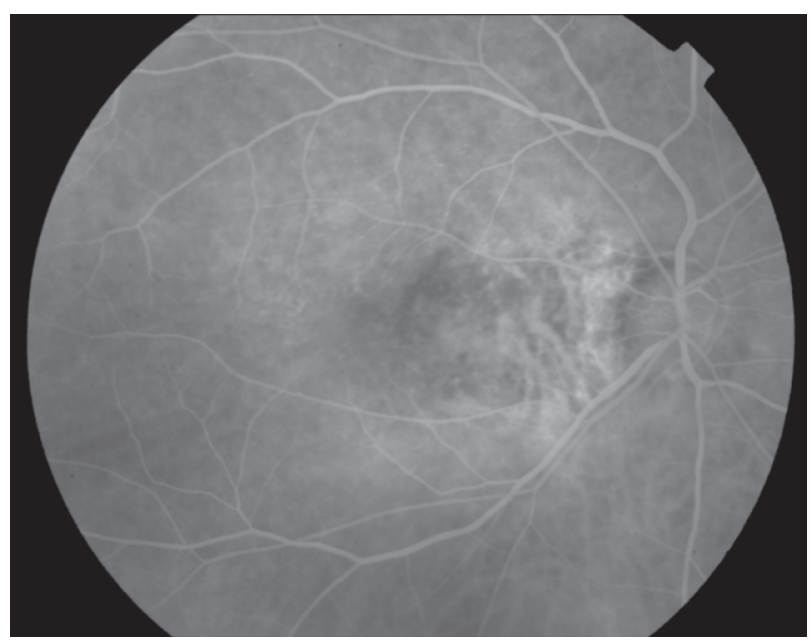

B

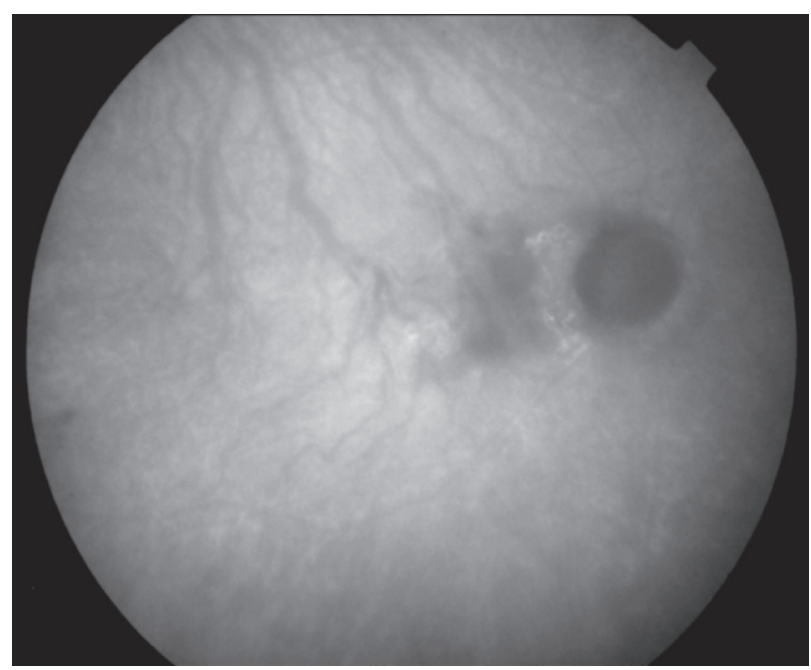

Figure 4 Six months after PDT late-phase FA A) and ICG B) show absence of leakage and complete disappearance of the CNV.

Abbreviations: CNV, choroidal neovascularization; FA, fluorescein angiography; PDT, photodynamic therapy.

where anti-VEGF treatment is not available, an adjustment of the PDT spot size to include the detection by ICG feeding complex could be an additional option in the treatment of the subfoveal CNV and might be considered as an alternative treatment.

\section{Disclosure}

The authors report no conflicts of interest in this work.

\section{References}

1. Photodynamic therapy of subfoveal choroidal neovascularization in age-related macular degeneration with verteporfin: one-year results of 2 randomized clinical trials-TAP report. Treatment of age-related macular degeneration with photodynamic therapy (TAP) Study Group. Arch Ophthalmol. 1999;117:1329-1345. 
2. Group TS. Photodynamic therapy of subfoveal choroidal neovascularization in age?related macular degeneration with verteporfin: Two-years results of 2 randomized clinical trials-TAP report 2. Arch Ophthalmol. 2001;119:198-207.

3. Kozak I, Cheng L, Cochran DE, Freeman WR. Phase I clinical trial results of verteporfin enhanced feeder vessel therapy in subfoveal choroidal neovascularisation in age related macular degeneration. $\mathrm{Br}$ J Ophthalmol. 2006;90:1152-1156.

4. Piermarocchi S, Lo Giudice G, Sartore M, et al. Photodynamic therapy increases the eligibility for feeder vessel treatment of choroidal neovascularization caused by age-related macular degeneration. Am J Ophthalmol. 2002;133:572-575.

5. Staurenghi G, Orzalesi N, La Capria A, Aschero M. Laser treatment of feeder vessels in subfoveal choroidal neovascular membranes: a revisitation using dynamic indocyanine green angiography. Ophthalmology. 1998;105:2297-2305

6. Staurenghi G, Massacesi A, Musicco I, Salvetti P, Viola F. Combining photodynamic therapy and feeder vessel photocoagulation: A pilot study. Semin Ophthalmol. 2001;16:233-236.

7. Fine HF. Photodynamic therapy in the anti-VEGF era. Br JOphthalmol. 2007;91:707-708.

8. Brown DM, Regillo CD. Anti-VEGF agents in the treatment of neovascular age-related macular degeneration: applying clinical trial results to the treatment of everyday patients. Am J Ophthalmol. 2007;144:627-637.
9. Pieramici DJ, Rabena MD. Anti-VEGF therapy: comparison of current and future agents. Eye. 2008;22:1330-1336.

10. Costa RA, Jorge R, Calucci D, Melo LA Jr, Cardillo JA, Scott IU. Intravitreal bevacizumab (Avastin) in combination with verteporfin photodynamic therapy for choroidal neovascularization associated with age-related macular degeneration (IBeVe Study). Graefes Arch Clin Exp Ophthalmol. 2007;245:1273-1280.

11. Ahmadieh H, Taei R, Soheilian M, Riazi-Esfahani M, Ahadi H. Singlesession photodynamic therapy combined with intravitreal bevacizumab for neovascular age-related macular degeneration. Eur J Ophthalmol. 2008;18:297-300

12. Miller JW, Schmidt-Erfurth U, Sickenberg M, et al. Photodynamic therapy with verteporfin for choroidal neovascularization caused by age-related macular degeneration: results of a single treatment in a phase 1 and 2 study. Arch Ophthalmol. 1999;117:1161-1173.

13. Schmidt-Erfurth U, Michels S, Barbazetto I, Laqua H. Photodynamic effects on choroidal neovascularization and physiological choroid. Invest Ophthalmol Vis Sci. 2002;43:830-841.

14. Sickenberg M, Ballini JP, van den Bergh H. [Visudyne photodynamic therapy and feeder vessel occlusion: rationale of a synergistic association and clinical options]. J Fr Ophtalmol. 2004;27:93-102. 